\title{
Neuer Schwung in der Debatte
}

\section{Eine jährlich um sieben Prozent ansteigende Energiesteuer soll den Umweltver- brauch belasten und gleichzeitig zur Senkung der Lohnnebenkosten beitragen. Mit diesem Konzept will der Deutsche Naturschutzring die erlahmte Diskussion um eine ökologische Steverreform wieder in Schwung bringen.}

$\mathrm{A}$ Von Danyel Reiche ufmerksame Beobachter der Debatte um eine Ökologische Steuerreform (ÖSR) fühlen sich an eine bekannte Geschichte erinnert. Everybody, somebody und nobody sollen ein wichtiges Problem angehen. Da sagt everybody, somebody solle beginnen. Somebody wiederum sagt, er werde nur aktiv, wenn everybody mitmache. So handelt am Ende nur nobody.

Die ÖSR-Diskussion ist bislang nach einem ähnlichen Strickmuster verlaufen. Zwar haben sich alle Parteien für eine Ökologische Steuerreform ausgesprochen. Auch Umweltminister Klaus Töpfer und seine Nachfolgerin Angela Merkel haben sich wiederholt für mehr Marktwirtschaft in der Umweltpolitik stark gemacht. Doch die Bundesregierung, nicht müde, Pest und Cholera für den "Standort Deutschland“ heraufzubeschwören, blockiert den Einstieg in eine Ökologische Steuerreform und hat die Verantwortung nach Europa geschoben. Die EU wiederum hat zwar ein ausgearbeitetes Konzept in der Schublade, dieses aber unter den Vorbehalt gestellt, daß es von allen OECD-Staaten mitgetragen werden müsse. So handelt am Ende nur nobody ... Geht es nach dem Deutschen Naturschutzring (DNR), soll die Tatenlosigkeit der Bundesregierung ein Ende haben. Die Dachorganisation der deutschen Natur- und Umweltschutzverbände hat jetzt ihr Konzept für eine Ökologische Steuerreform vorgestellt. Von Oktober 1996 an haben Experten aus den über 100 DNR-Mitgliedsverbänden - dazu zählen große Organisationen wie BUND und NABU ebenso wie etwa die Aktion Fischotterschutz und der Deutsche Kanuverband - mit externem Sachverstand an ihrem „Positionspapier Ökologische Steuerreform" gefeilt, ehe die unterschiedlichen Interessen unter einen Hut gebracht werden konnten. „Daß wir es geschafft haben, eine derart große Anzahl an Organisationen und divergierenden Ansichten bei einem so sensiblen Thema zusammenzubringen, darf man ohne große
Umschweife als sensationell bezeichnen“, sagt Martin Rocholl, der das DNR-Projekt koordiniert hat. Damit, so Rocholl, dürfte die Ökosteuer-Debatte noch einmal an Schwung gewinnen.

\section{- Umweltverbrauch belasten, Arbeitskosten entlasten}

Im einzelnen haben sich die Verbände auf ein Konzept verständigt, das unter dem griffigen Slogan „Umweltverbrauch belasten, Arbeitskosten entlasten“ steht. Zunächst soll eine jährlich um sieben Prozent ansteigende Energiesteuer eingeführt werden. Die Höhe der Energiesteuer, heißt es, sei so gewählt, daß einerseits deutliche Anreize zum Energiesparen geschaffen werden, andererseits aber genug Zeit für die Wirtschaft bleibe, sich anzupassen. Da der Steigerungspfad der Steuer langsam und kalkulierbar verlaufe, könnten sich Unternehmer und Verbraucher rechtzeitig umstellen und entsprechende Energiesparinvestitionen in die Wege leiten.

Das Aufkommen aus der Energiesteuer soll vorrangig zu einer Senkung der Lohnnebenkosten eingesetzt werden. Damit werde gleichzeitig ein positives Signal für mehr Beschäftigung gesetzt. Der arbeitsintensiven ökologischen Landwirtschaft etwa könne damit ebenso zum Durchbruch verholfen werden wie erneuerbaren Energien, die endlich konkurrenzfähig würden. Die Ökologische Steuerreform sei also Lösung für zwei drängende Probleme: die Umweltkrise mit drohendem Klimakollaps, Müllawine, Ozonloch und Artenschwund, sowie die Beschäftigungskrise mit Arbeitslosenzahlen auf Rekordniveau.

Zusätzliche Abgaben sieht das Konzept im Verkehrsbereich vor. Um die Blechlawine zurückzudrängen, sei eine weitergehende Besteuerung des Straßenverkehrs unabdingbar, heißt es in dem Positionspapier. Deshalb soll die Mineralölsteuer um jährlich 30 Pfennig pro Liter steigen. Für den Luftverkehr als ,umweltschädlich- ste Form der Fortbewegung" fordert der DNR, endlich die Steuerbefreiung für Flugtreibstoffe aufzuheben.

\section{- Vorreiter Dänemark im Wachstumsboom}

Daß eine Ökologisch Steuerreform keineswegs mit gesamtwirtschaftlichen Nachteilen verbunden sein muß, sondern die Volkswirtschaft vielmehr sogar beleben kann, zeigen die Niederlande, Schweden und Dänemark, die nicht mehr auf die schleppenden Diskussionen auf EU-Ebene warten mochten und in Sachen Ökologische Steuerreform mutig vorangeschritten sind. ÖSRVorreiter Dänemark, das seine 1994 eingeführten Umweltabgaben noch bis zum Jahr 2000 jährlich erhöhen will, konnte nicht zuletzt durch einen Boom in der Windenergiebranche seine Arbeitslosigkeit um ein Drittel von 12,5 (1993) auf 8,7 Prozent (1997) senken. Die Wachstumsraten zählen zu den höchsten in der OECD, nächstes Jahr kann das Land sogar erstmals einen Haushaltsüberschuß erwirtschaften.

„Angesichts der Erfahrungen unserer Nachbarstaaten ist es unverständlich, warum die Bundesregierung die Ökologische Steuerreform nicht als Chance für Umweltschutz und Wirtschaft begreift", betont DNR-Projektleiter Rocholl. Wenn die Bundesregierung ihr eigenes Klimaschutzziel aber noch ernst nehme und zugleich nicht den Anschluß bei der Entwicklung umweltverträglicher Technologien verpassen wolle, sei jetzt dringend entschlossenes Handeln geboten.

\section{Anmerkung}

Das DNR-Positionspapier zur Ökologischen Steverreform kann gegen zwei Mark in Briefmarken bestellt werden bei der DNR-Koordinationsstelle Ökologische Finanzreform, Dr. Martin Rocholl, Am Dorfbach 16, 79111 Freiburg,

Tel./Fax (0761) 472960.

e-mail: m.rocho|@oln.comlink.apc.org

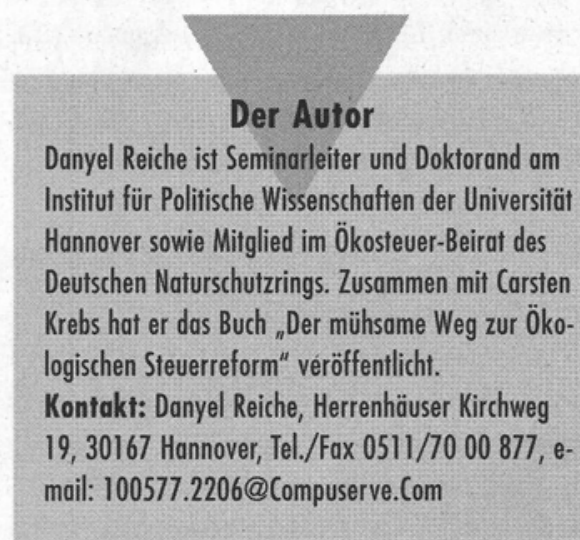


(c) 20I0 Authors; licensee IÖW and oekom verlag. This is an article distributed under the terms of the Creative Commons Attribution Non-Commercial No Derivates License (http://creativecommons.org/licenses/by-nc-nd/3.o/), which permits unrestricted use, distribution, and reproduction in any medium, provided the original work is properly cited. 